\title{
Difference in Aortic Valve Area Measured With Cardiac CT and Transthoracic Echocardiography
}

\author{
Sung Min Ko, MD'1, Jose F. Condado, MD², Vasilis Babaliaros, MD², Stamatios Lerakis, MD², \\ Yi-An Ko, PhD ${ }^{3}$, Arthur E. Stillman, MD ${ }^{*}$ \\ ${ }^{1}$ Department of Radiology, Konkuk University Medical Center, Seoul, Korea \\ 2 Department of Cardiology, Emory University Hospital, Atlanta, Georgia, United States \\ ${ }^{3}$ Department of Biostatistics and Bioinformatics, Emory University Hospital, Atlanta, Georgia, United States \\ ${ }^{4}$ Department of Radiology, Division of Cardiothoracic Imaging, Emory University Hospital, Atlanta, Georgia, United States
}

\section{Abstract}

Background: There is difference in aortic valve area (AVA) measurement between cardiac computed tomography (CCT) and transthoracic echocardiography (TTE).

Objectives: To evaluate factors affecting the measurement of AVA obtained with CCT and TTE in patients with severe aortic stenosis.

Method: One hundred twenty-seven consecutive patients (median age, 81 years, 57\% women) that underwent TTE, CCT, and transcatheter aortic valve replacement were included. AVA was deduced from the continuity equation on TTE (AVATTE) and manual planimetry on CCT (AVACCT). Factors that related to difference between AVACCT and AVATTE were evaluated by linear regression analysis.

Result: AVACCT $(0.92 \pm 0.36 \mathrm{~cm} 2, p<0.001)$ was significantly greater than AVATTE $(0.69 \pm 0.16 \mathrm{~cm} 2)$. There was a weak positive correlation between AVAs measured with CCT and TTE $(r=0.25, p=0.004)$. There was significant difference between CCT $(5.0 \pm 0.92 \mathrm{~cm} 2, p<0.001)$ and TTE $(3.52 \pm 0.77 \mathrm{~cm} 2)$ measurements of left ventricular outflow tract (LVOT) area. The LVOT area was generally elliptical ( $>10 \%$ difference between LVOT diameters in $\mathbf{9 5 . 3 \%}$ patients). Multiple linear regression

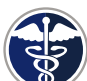

Fax +1 2037853346

E-Mail: jshd@scienceinternational.org

http://structuralheartdisease.org/

\author{
(C) 2019 Journal of Structural Heart Disease \\ Published by Science International Corp. \\ ISSN 2326-4004 \\ Accessible online at: \\ http://structuralheartdisease.org/
}

showed that difference between AVACCT and AVATTE was significantly associated with log-transformed aortic valve calcium score (estimate $-0.267, p<0.001$ ), LVOT area difference between CCT and TTE (estimate $-0.082, p=0.006$ ), and age (estimate $-0.006, p=0.01$ ).

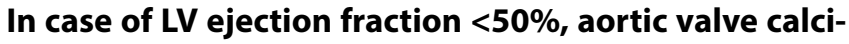
um score $\geq 1,651$, LVOT eccentricity $\geq 0.78$, presence of atrial fibrillation, absence of significant calcification of aortic valve, or mean transaortic pressure gradient $\leq \mathbf{4 0}$ $\mathrm{mmHg}$, there was no significant correlation between AVACCT and AVATTE.

Conclusion: Age, Agatston aortic valve score, and LVOT area difference between CCT and TTE might affect difference between AVACCT and AVATTE in patients with severe aortic stenosis.

Copyright @ 2019 Science International Corp.

\section{Key Words}

Computed tomography - Echocardiography - Aortic stenosis • Aortic valve area • Planimetry

\section{Introduction}

Aortic stenosis (AS) is one of the most common valvular heart disease (VHD) worldwide. Its prevalence is increased with advancing age. In addition, AS is

\footnotetext{
* Corresponding Author:

Arthur E. Stillman, MD

Department of Radiology, Division of Cardiothoracic Imaging

Emory University Hospital

1364 Clifton Road Northeast, , , Atlanta, GA 30322

Tel. +404 712 7964; Fax: +404 712 7777; E-Mail: aestill@emory.edu
} 
the most common VHD requiring valve replacement in Europe and North America [1, 2]. Its clinical importance has increased health care expenditure and caused exponential growth in the application of transcatheter aortic valve replacement (TAVR) [3].

Echocardiography and computed tomography (CT) are complementary imaging techniques for TAVR. They are used to evaluate patient selection and optimal transcatheter valve size selection in patients with symptomatic severe AS and degenerative tricuspid valve [4-6]. Appropriate patient selection based on clinical symptoms and the severity of AS is of maximal importance for successful TAVR procedure [7]. The aortic valve area (AVA) is an important and widely used parameter to determine AS hemodynamic severity. It is traditionally calculated at Doppler transthoracic echocardiography (TTE) by using the continuity equation (AVA $\mathrm{ATE}_{\mathrm{TE}}$ ). It is considered as the major independent predictor of outcome in AS $[8,9]$. Cardiac CT (CCT) using multiphase reconstruction of the cardiac cycle can provide imaging of aortic valve motion. Measurement of AVA can be obtained using direct planimetry on CCT images (AVAccT) [10]. However, there is no objective non-invasive reference standard to determine true AVA in patients with severe AS. The functional AVA or AVA $A_{T T E}$ can significantly underestimate AVA because the left ventricular outflow tract (LVOT) area is underestimated by using a single-diameter measurement assuming circular geometry [11]. Most frequently, the LVOT is ellipsoid. The anatomical AVA or AVA CcT $_{\text {is }}$ is larger than AVA TTE. $_{\text {. }}$ Several factors such as aortic valve calcification and LVOT morphology can affect AVA or AVAcct [12-15].

No studies have assessed factors affecting measurement differences of AVA between TTE and CCT. We hypothesize that the different size of LVOT measured with CCT and TTE and shape (or eccentricity) of LVOT obtained by CCT are associated with differences of AVA measured with TTE and CCT. However, other variables might significantly affect this difference. This may have important clinical implication in calculating AVA in patients with severe AS. Thus, the aim of this study was to identify factors affecting the difference between AVA measured by planimetry on CCT and AVA obtained by continuity equation on TTE in patients with symptomatic severe AS.

\section{Material and Methods}

\section{Study Population}

Patients were drawn from a single-center study of patients who underwent balloon expandable TAVR (SAPEIN and SAPIEN XT, Edwards Lifescience, Irvine, CA, USA) from January $1^{\text {st }}, 2013$ to November $30^{\text {th }}$, 2014. The inclusion criteria were: patients who had severe AS (defined as AVA $<1 \mathrm{~cm}^{2}$, mean transvalvular gradient $>40 \mathrm{mmHg}$, or peak transvalvular velocity $>4 \mathrm{~m} / \mathrm{s}$ or any combination) [16], with New York Heart Association (NYHA) class II, III, or IV heart failure symptoms, and with high surgical risk based on the Society for Thoracic Surgeons (STS) risk score. The exclusion criteria were: patients who needed valvein-valve procedures and those who had previous mitral valve replacement. TTE and CCT were performed within 4 weeks without interval change in clinical status or cardiovascular event. This retrospective study was approved and performed in accordance with the regulations of the hospital Institutional Review Board. All patients gave written informed consent before participation.

\section{Transthoracic echocardiography}

A single highly experienced operator performed TTE in all patients using VIVID 7 ultrasound machine (General Electric, Milwaukee, WI, USA). Collected data were as follows: maximal blood flow velocities at aortic valve and LVOT, time velocity integrals at aortic valve and LVOT, LVOT diameter, and AVA obtained from the continuity equation $\left[\pi \times(\text { LVOT diameter } / 2)^{2}\right.$ $\times$ (velocity time integral of the LVOT/velocity time integral of the transaortic flow)]. Mean and maximal transvalvular aortic pressure gradients were recorded.

\section{Cardiac Computed Tomography Examination}

All ECG-gated contrast-enhanced CCT examinations were performed using a dual-source CT scanner (Somatom Definition, Siemens Medical Solutions, Forchheim, Germany). Data acquisition was performed in a craniocaudal direction with detector collimation of $2 \times 32 \times 0.6 \mathrm{~mm}$, slice acquisition of $2 \times$ $64 \times 0.6 \mathrm{~mm}$, gantry rotation time of $330 \mathrm{~ms}$, pitch of $0.20-0.43$ adapted to HR, tube voltages of $120 \mathrm{kV}$ for calcium scoring and CCT, tube current-time product of 100-140 mAs per rotation for calcium scoring, and 
100-280 mAs per rotation for CCT. A non-enhanced electrocardiography (ECG)-gated CT scan prospectively triggered at $75 \%$ of the R-R interval was performed to measure the aortic valve calcium score. For CCT, ECG-based tube current modulation was not implemented. Contrast agent application was controlled by a bolus tracking technique using 80 to $120 \mathrm{ml}$ of contrast media (Isovue-370, lopamidol Injection 76\%, Bracco Diagnostics, Inc). Ten transaxial data sets were reconstructed with retrospective ECG gating at $10 \%$ steps from $0-90 \%$ of the R-R interval for each patient.

\section{CCT image analysis}

All data were transferred to a dedicated workstation (Syngo Via software, Siemens Medical Solutions, Forchheim, Germany). Analysis of CCT images was performed by a cardiac radiologist (13 years of experience with CCT) who was blinded to patient clinical data including all clinical findings, history, and TTE results. Repeat assessments were performed by the same radiologist at least 1 month apart in random order to prevent recall bias.

The AVA was measured by planimetry of the smallest area of the aortic valve opening on the time point of maximal aortic valve opening (early or mid-systole, $10 \%-20 \%$ of the R-R interval), using oblique coronal and oblique sagittal planes along the LVOT and an additional oblique transverse plane parallel to the aortic valve. The largest cross-sectional area of the LVOT was measured at the hinge point of the insertion of 3 aortic cusps on the double-oblique transverse plane during mid-systole ( $20 \%$ of the R-R interval). The anatomic AVA and LVOT area were calculated as average of 2 planimetric measurements using an electronic caliper [13]. The following measures were obtained: LVOT minimal and maximal diameters ( $D_{\min }$ and $\left.D_{\max }\right)$ and LVOT area excluding aortic annulus calcification (Figure 1) [17]. The eccentricity index of LVOT was determined as $D_{\min } / D_{\max }$. LVOT was considered as circular if the index was greater than 0.9 [13].
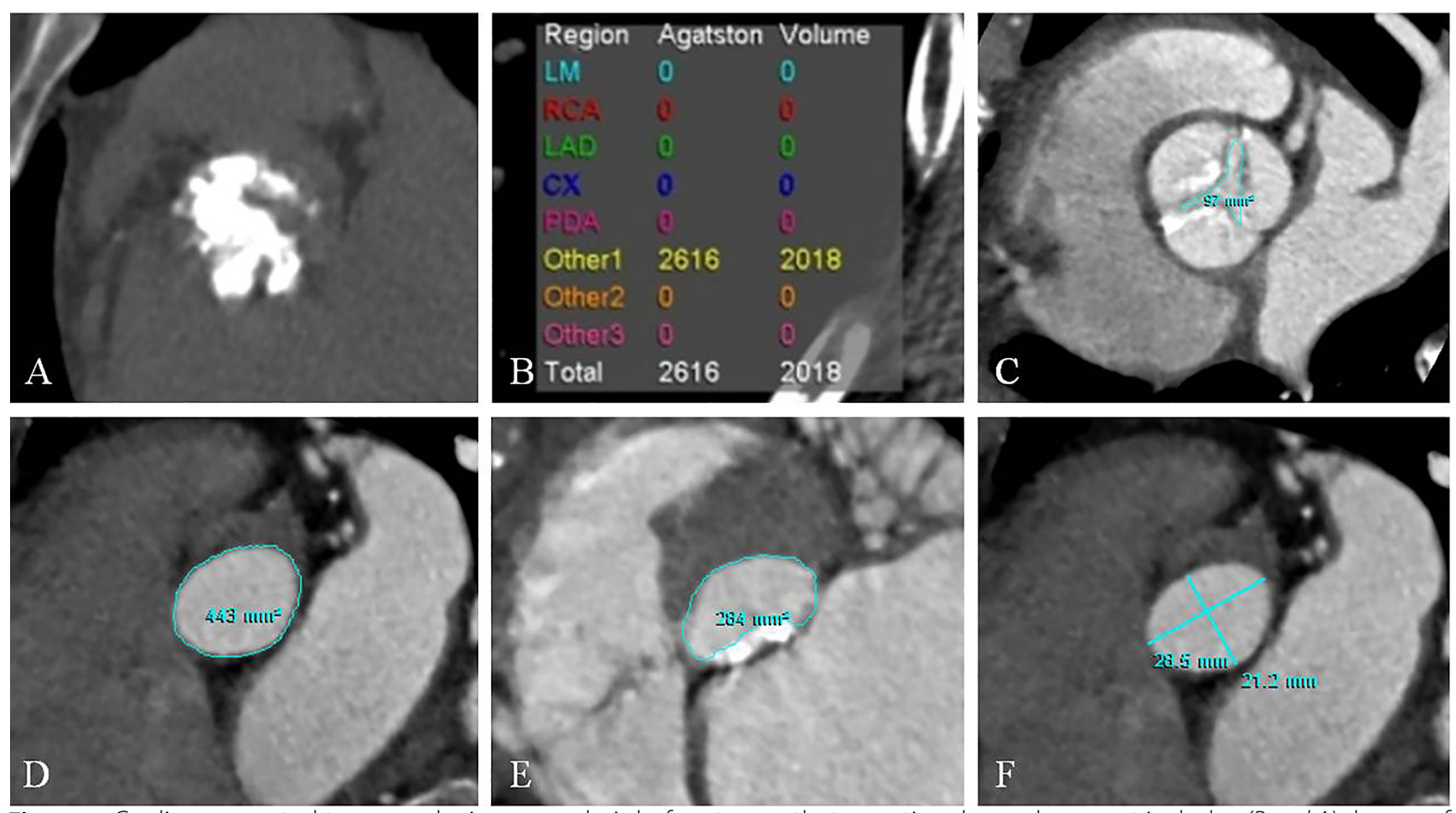

Figure 1. Cardiac computed tomography image analysis before transcatheter aortic valve replacement includes (Panel A) degree of aortic valve calcification and Agatston aortic valve score, (Panel B) aortic valve area (AVA) measurement, (Panel C) left ventricular outflow tract (LVOT) area measurement without calcification, (Panel D) LVOT area measurement with calcification, and (Panel E) minimum (Dmin) and maximum (Dmax) diameters of LVOT. 
Table 1. Baseline characteristics

\begin{tabular}{|c|c|}
\hline & All $(n=127)$ \\
\hline Age (years) & $81(74,85)$ \\
\hline Female & $72(56.6)$ \\
\hline Caucasian & $112(88.8)$ \\
\hline $\mathrm{BMI}\left(\mathrm{kg} / \mathrm{m}^{2}\right)$ & $26.2(22.8,29.4)$ \\
\hline STS PROM score (\%) & $8.9(5.9,11.8)$ \\
\hline Diabetes & $55(43.3)$ \\
\hline Dyslipidemia & $119(93.7)$ \\
\hline Hypertension & $119(93.7)$ \\
\hline $\begin{array}{l}\text { Chronic Lung Disease } \\
\text { None } \\
\text { Mild } \\
\text { Moderate } \\
\text { Severe }\end{array}$ & $\begin{array}{l}56(44.1) \\
24(18.9) \\
16(12.6) \\
31(24.4)\end{array}$ \\
\hline Coronary Artery Disease & $73(57.4)$ \\
\hline Prior $C A B G$ & $38(30.0)$ \\
\hline Prior cerebrovascular disease & $32(25.2)$ \\
\hline Peripheral Vascular Disease & $48(37.8)$ \\
\hline Immunocompromised & $29(22.9)$ \\
\hline Atrial fibrillation & $54(42.5)$ \\
\hline ESKD on dialysis & $4(3.1)$ \\
\hline Creatinine & $1.0(0.8,1.2)$ \\
\hline $\begin{array}{l}\text { Mitral Regurgitation } \\
\text { None/trace } \\
\text { Mild } \\
\text { Moderate } \\
\text { Severe }\end{array}$ & $\begin{array}{c}36(28.3) \\
51(40.2) \\
31(24.4) \\
9(7.1)\end{array}$ \\
\hline $\begin{array}{l}\text { Aortic Regurgitation } \\
\text { None/Trace } \\
\text { Mild } \\
\text { Moderate } \\
\text { Severe }\end{array}$ & $\begin{array}{c}50(39.4) \\
53(41.7) \\
20(15.7) \\
4(3.1)\end{array}$ \\
\hline
\end{tabular}

Values are number (\%) or median (Q1, Q3). BMI = body mass index; $C A B G=$ coronary artery bypass grafting; $E F=$ ejection fraction; $G F R=$ glomerular filtration rate; $\mathrm{AV}=$ aortic valve; $\mathrm{LVOT}=$ left ventricular outflow track; STS-PROM = Society of Thoracic Surgery Predicted Risk of Mortality; ESKD = end-stage kidney disease

CCT image quality was classified using a 4-point subjective ranking scale as follows: (1) bad; (2) poor, but diagnostic; (3) good; and (4) excellent. Aortic valve Agatston calcium score was evaluated using Syngo Via software (Siemens Medical Solutions, Forchheim, Germany). The aortic valve calcification grade was categorized as absent, mild, moderate, or severe as described by Willmann et al [18]. We only assessed the degree of aortic annular calcification according to a previously described semiquantitative classification [19].

\section{Statistical analysis}

Quantitative variables are expressed as median and interquartile range (IQR) or mean and standard deviation. Categorical variables are expressed as number and percentage. The means of quantitative variables were compared with each other using Student's t-test. Intra-class correlation coefficient was used to investigate intra-observer and inter-observer agreement. For inter-observer agreement, we used previous radiologists' reports and measurements by one observer. Pearson correlation coefficient was used to assess the correlation between CCT and TTE measurements of AVA in the whole cohort and various predefined subgroups. Bland-Altman method was used to study the variability of methods used for measuring AVA and LVOT area. Multivariate analysis was performed using a linear regression model by including the difference in AVAs measured by CCT and TTE as response variable and covariates. Associations of difference between AVAcct and AVA TTE with $_{\text {. }}$ different variables were evaluated by multiple linear regression analysis. $P$-values less than 0.05 were considered statistically significant. All statistical analysis and related graphics were performed using SAS 9.4, IBM SPSS statistics (IBM, Armonk, NY, USA).

\section{Results}

\section{Patients}

The median age of the population was 81 years (IQR: 11$)$. The majority (57\%) of these patients were females. Patient characteristics of the study cohort are summarized in Table 1. The median calculated STS Predicted Risk of Mortality score was $8.9 \%$ (IQR: 5.9). In terms of comorbidity, prevalence of hypertension (93.7\%), diabetes (43.3\%), coronary artery disease (57.4\%), chronic pulmonary disease (55.9\%), and atrial fibrillation (AF, 42.5\%) were high. The CCT image quality was assessed to be excellent or good in $103(81 \%)$ cases and poor but evaluable in $24(19 \%)$ cases. Aortic valve calcification was grade 1 in $1(1 \%)$ 

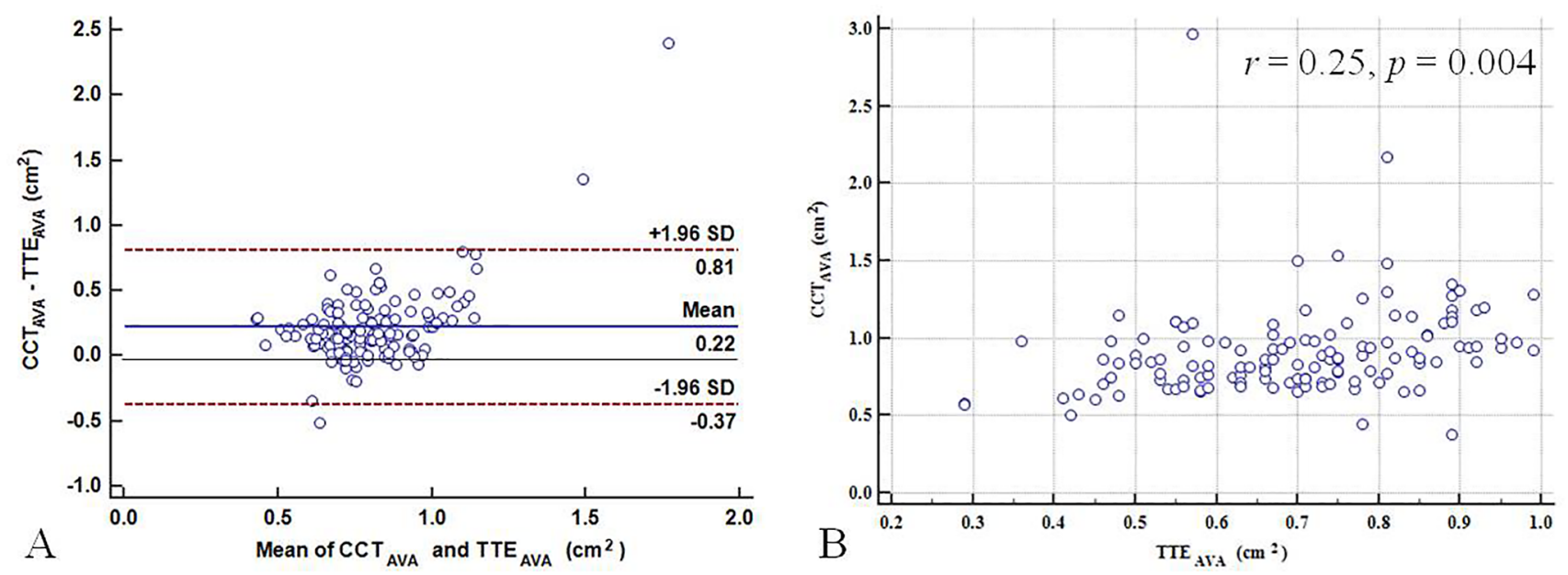

Figure 2. Panel A. Bland-Altman plot. Panel B. Pearson correlation of cardiac computed tomography (CCT) and transthoracic echocardiography (TTE) measurements of the aortic valve area (AVA).

patient, grade 2 in 28 (22\%) patients, grade 3 in 63 (50\%) patients, grade 4 in 35 (27\%) patients. Median aortic valve calcium score was 845 (IQR: 916) Agatston units. There was a strong correlation $(r=0.74, p$ $<0.0001$ ) between the grade of aortic valve calcification and aortic valve Agatston calcium score. Aortic annular calcification was grade 1 in 33 (26\%) patients, grade 2 in $36(28 \%)$ patients, and grade 3 in $5(2 \%)$ patients. There was a moderate correlation $(r=0.44$, $p<0.0001)$ between the grade of aortic annular calcification and aortic valve Agatston calcium score or between the grade of aortic valve and aortic annular calcification $(r=0.33, p=0.0001)$. All patients were in NYHA functional class III/IV. They were high surgical risk patients.

\section{Assessment of AVA and LVOT structure}

AVA measured with CT planimetry (mean, $0.91 \pm$ $0.30 \mathrm{~cm}^{2}$ ) was significantly greater than that computed with TTE measurements (mean, $0.69 \pm 0.16 \mathrm{~cm}^{2}$; $p<0.001$ ) (Figure 2A). There was a weak positive correlation between AVA $_{\text {CCT }}$ and AVA $A_{\text {TTE }}(r=0.25, p=$ 0.004) (Figure 2B). Of our 127 patients who had an AVA $_{\text {TTE }}$ of $<1.0 \mathrm{~cm}^{2}, 31$ (24\%) patients had an AVAccT of $>1.0 \mathrm{~cm}^{2}$ and would be reclassified to moderate AS $(n=28)$ or mild AS $(n=1)$ or no AS $(n=2)$ by AVAccr. Mean LVOT diameter on TTE was $2.11 \pm 0.25 \mathrm{~cm}$. The mean minimal diameter of LVOT measured by CCT was $2.24 \pm 0.27 \mathrm{~cm}$. A significant correlation was

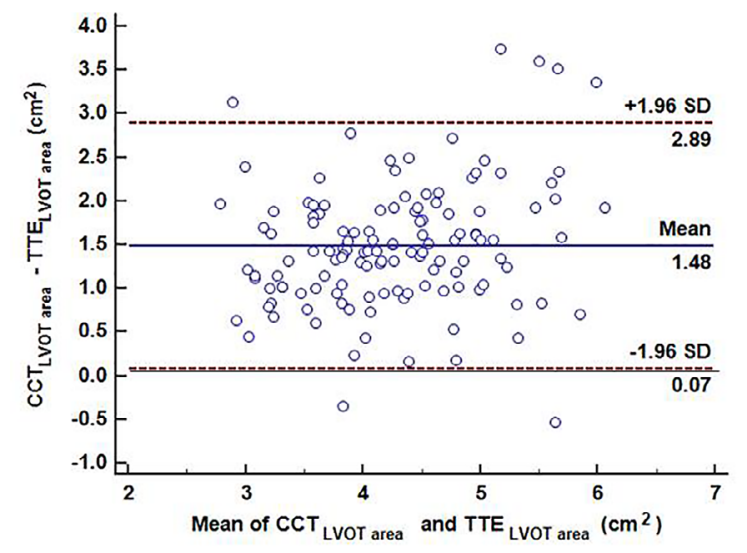

Figure 3. Bland-Altman plot of cardiac computed tomography (CCT) and transthoracic echocardiography (TTE) measurements of the left ventricular outflow tract (LVOT) area.

found between these two values $(r=0.65 ; p<0.0001)$, with a mean difference of $0.14 \mathrm{~cm}$ (95\% confidence interval [Cl]: 0.10 to $0.18 \mathrm{~cm}$ ). There was a significant difference between CCT (mean, $5.0 \pm 0.92 \mathrm{~cm}^{2}$ ) and TTE (mean, $3.52 \pm 0.77 \mathrm{~cm}^{2} ; p<0.001$ ) measurements for the LVOT area (Figure 3). There was a good correlation between LVOT area measured with CCT and TTE $(r=0.65 ; p<0.0001)$. Evaluation by CCT showed that the LVOT area was generally elliptical (95.3\% patients), with an eccentricity index of $0.78 \pm 0.07$ in the entire cohort (Figure 4) (Table 2). Intra-class correlation coefficient for intra-observer measurements of 

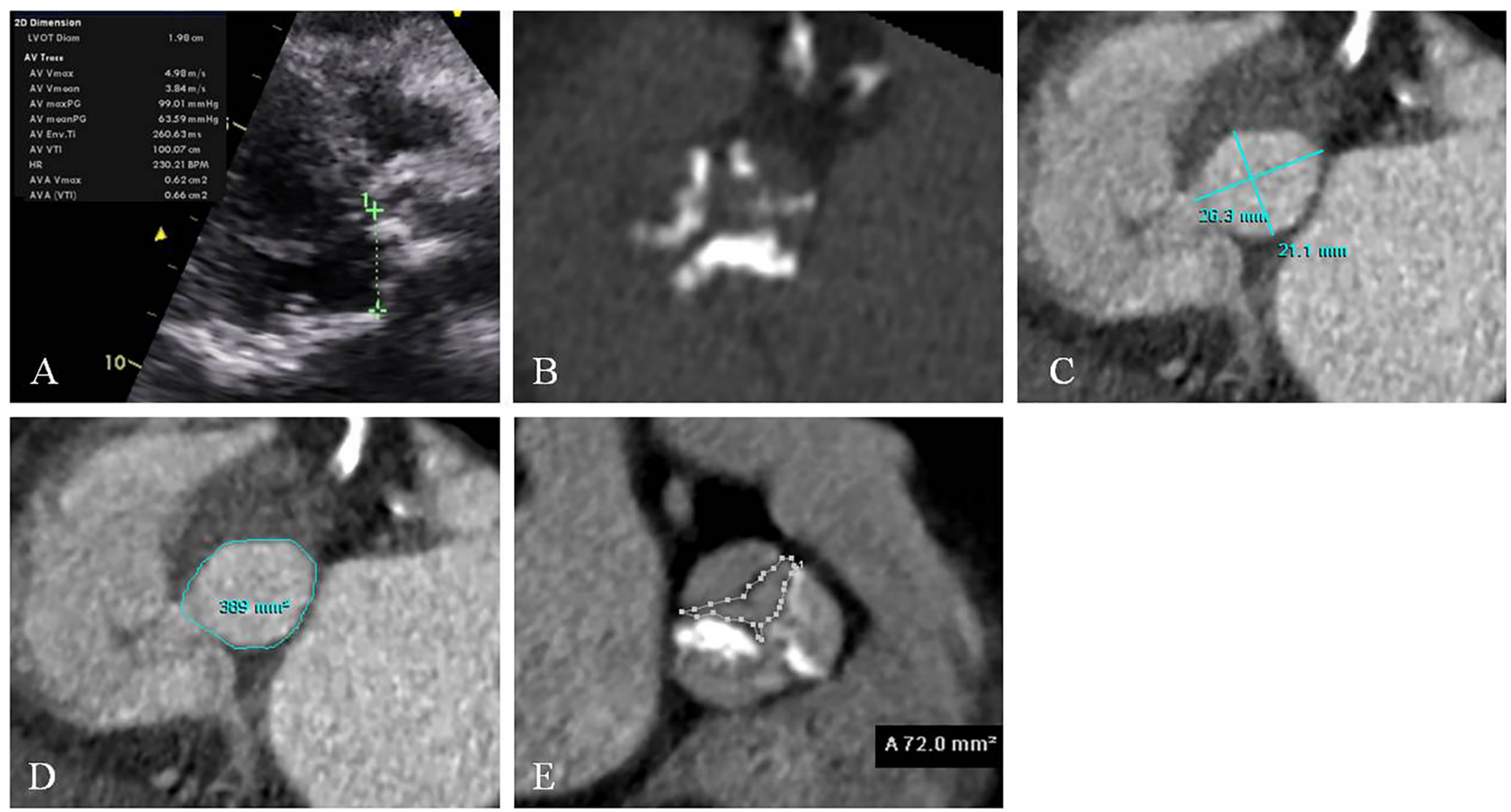

Figure 4. Images for a 68-year-old woman with severe aortic stenosis. Ejection fraction was 59\%. Panel A. On transthoracic echocardiography, left ventricular outflow tract (LVOT) diameter was measured as $1.98 \mathrm{~cm}$ and LVOT area was calculated as $3.10 \mathrm{~cm} 2$. Aortic valve area (AVA) was measured as $0.66 \mathrm{~cm} 2$ by using the continuity equation. Panel $B$. Severe degree of aortic valve calcification on pre-contrast cardiac computed tomography (CCT) image. Agatston aortic valve score was 2,101. Panel C. The LVOT was elliptical on multiplanar reformatted CCT with an eccentricity index of 0.80 measured as the ratio of the minimum $(2.11 \mathrm{~cm}) / \mathrm{maximum}$ diameter $(2.63 \mathrm{~cm})$. Panel D. The measured LVOT area was $3.89 \mathrm{~cm} 2$. Panel E. CCT planimetry AVA was $0.72 \mathrm{~cm} 2$.

AVAcct and LVOT area measured with CCT were 0.973 (95\% Cl: 0.963 to 0.981$)$ and 0.948 (95\% Cl: 0.927 to 0.963), respectively. Intra-class correlation coefficient for inter-observer measurements of AVAcct and LVOT area measured with CCT were 0.60 (95\% Cl: 0.38 to 0.74 ) and 0.90 ( $95 \% \mathrm{Cl}: 0.86$ to 0.93$)$, respectively.

\section{Assessment of difference between AVAcct and AVA $A_{T T E}$}

Simple linear regression analysis showed that difference between AVA cct $_{\text {and }}$ AVA $A_{\text {TTE }}$ was associated with age, aortic valve calcification grade (0/1 vs. $2 / 3)$, log-transformed aortic valve calcium score, transvalvular mean pressure gradient, CCT image quality, and LVOT area difference between CCT and TTE. Multiple linear regression analysis revealed that the following three variables were significantly associated with difference between AVA $A_{\text {cct }}$ and AVA $A_{T T E}$ : log-transformed aortic valve calcium score (inverse relationship), LVOT area difference between CCT and TTE, and age (inverse relationship) in decreasing order of significance
(Table 3). On subgroup analysis, AVAs measured with CCT were not correlated with AVA by TTE in group with LVEF $<50 \%$, aortic valve calcium score $>1,651$, LVOT eccentricity $\geq 0.78$, presence of $A F$, absence or mild grade of aortic valve calcification, or transvalvular pressure gradient $\leq 40 \mathrm{mmHg}$ (Table 4).

\section{Discussion}

This study demonstrated that a discrepancy between CCT and TTE measurements of AVA that was significantly associated with log-transformed Agatston aortic valve score, LVOT area difference measured with CCT and TTE, and age in patients with severe AS. However, LVOT eccentricity and aortic annular calcification severity were not associated with difference between AVAcct and AVA

The hemodynamic (AVA $A_{T T E}$ ) and anatomic (AVA ${ }_{C C T}$ ) AVA are not interchangeable. Our result was consistent with previous studies showing that continuity 
Table 2. CCT and TTE measurements.

\begin{tabular}{lccc}
\hline Measurements & Mean \pm SD & Minimum & Maximum \\
\hline TTE & & & \\
AVA $\left(\mathrm{cm}^{2}\right)$ & $0.69 \pm 0.16$ & 0.29 & 0.99 \\
LVOT diameter (cm) & $2.11 \pm 0.25$ & 0.13 & 2.74 \\
LVOT area (cm $\left.{ }^{2}\right)$ & $3.52 \pm 0.77$ & 1.32 & 5.9 \\
LVEF (\%) & $51.2 \pm 14.3$ & 10 & 73 \\
Transaortic mean & $44.6 \pm 14.8$ & 18 & 110 \\
gradient (mmHg) & & & \\
CCT & & & \\
AVA (cm $\left.{ }^{2}\right)$ & $0.91 \pm 0.30$ & 0.38 & 2.97 \\
$\begin{array}{l}\text { LVOT minimum diame- } \\
\text { ter (cm) }\end{array}$ & $2.25 \pm 0.27$ & 1.57 & 3.20 \\
$\begin{array}{l}\text { LVOT maximum diam- } \\
\text { eter (cm) }\end{array}$ & $2.88 \pm 0.31$ & 2.15 & 3.83 \\
$\begin{array}{l}\left.\text { LVOT area (cm }{ }^{2}\right) \\
\text { LVOT eccentricity }\end{array}$ & $5.0 \pm 0.92$ & 3.24 & 7.66 \\
index & $0.78 \pm 0.07$ & 0.62 & 0.98 \\
\hline
\end{tabular}

$\mathrm{AVA}=$ aortic valve area; $\mathrm{TTE}=$ transthoracic echocardiography $; \mathrm{CCT}=$ cardiac computed tomography; LVEF = left ventricular ejection fraction; LVOT = left ventricular outflow track; TTE = transthoracic echocardiography

equation-derived $\mathrm{AVA}_{\mathrm{TTE}}$ was significantly smaller than planimetry-derived AVAcct, mainly due to the flow contraction phenomenon observed at the aortic valve [9-15]. In our study, $24 \%(n=31)$ of patients would be reclassified to moderate AS $(n=28)$ or mild AS $(n=1)$ or no AS $(n=2)$ by AVAcct. Therefore, a simple comparison between these two AVAs will thus be associated with difference and several individual factors have already been associated with this difference [12-15].

Underestimation of AVATTE may lead to discordance in AS severity grading, particularly for those with low mean pressure gradient $<40 \mathrm{mmHg}$ despite small AVA $<1 \mathrm{~cm}^{2}$ [20]. We have noted that most patients (95\%) had elliptical shape of LVOT and that LVOT diameter measured by TTE was smaller than LVOT minimum diameter measured by CCT. As a result, TTE significantly underestimated LVOT area and AVA when compared to CCT. Several studies have demonstrated that noncircular shape (ellipticity) of the LVOT and underestimation of LVOT area results in underestimation of the continuity equation-derived AVATTE by
Table 3. Factor affecting the difference in AVAs measured by CCT and TTE: simple and multiple linear regression analyses

\begin{tabular}{lccc}
\hline & $\begin{array}{c}\text { Parameter } \\
\text { estimate }\end{array}$ & $\begin{array}{c}\text { Standard } \\
\text { error }\end{array}$ & p-value \\
\hline $\begin{array}{l}\text { Simple linear regression } \\
\text { analysis }\end{array}$ & & & \\
Log10 AVC Agatston & -0.3095 & 0.0349 & $<0.001$ \\
score & & & \\
AVC grade (0/1 vs. 2/3) & -0.2396 & 0.0606 & $<0.001$ \\
Transvalvular mean PG & -0.1636 & 0.0545 & 0.003 \\
LVOT area difference & 0.0983 & 0.0368 & 0.009 \\
between CCT and TTE & & & \\
CCT image quality & -0.195 & 0.0667 & 0.004 \\
Age & -0.0082 & 0.0031 & 0.009 \\
Annular Ca 4 grade & -0.0858 & 0.0572 & 0.136 \\
Atrial fibrillation & 0.0708 & 0.0542 & 0.194 \\
LVOT eccentricity & -0.2236 & 0.3863 & 0.564 \\
Sex & -0.0262 & 0.0545 & 0.632 \\
LVEF & -0.0009 & 0.0019 & 0.648
\end{tabular}

Multiple linear regression analysis

$\begin{array}{lccc}\text { Age } & -0.0061 & 0.0024 & 0.013 \\ \begin{array}{l}\text { Log10 AVC Agatston } \\ \text { score }\end{array} & -0.2670 & 0.0394 & <0.001 \\ \begin{array}{l}\text { LVOT area difference } \\ \text { between CCT and TTE }\end{array} & 0.0822 & 0.0291 & 0.006 \\ \begin{array}{l}\text { Transvalvular mean PG } \\ \text { AVC grade (0/1 vs. 2/3) }\end{array} & -0.0286 & 0.0465 & 0.539 \\ \text { CCT image quality } & -0.1037 & 0.0553 & 0.063\end{array}$

AVA = aortic valve area; $A V C=$ aortic valve calcification; $C a$ = calcification; $C C T$ = cardiac computed tomography; LVEF = left ventricular ejection fraction; $\mathrm{LVOT}=$ left ventricular outflow track; $\mathrm{PG}=$ pressure gradient; TTE = transthoracic echocardiography

two-dimensional echocardiography when compared to three-dimensional echocardiography [21, 22]. We found that the numeric difference between AVA and AVATTE was increased with increasing difference in LVOT area between CCT and TTE. However, the CCT measurement of LVOT eccentricity was not associated with difference between AVAcct and AVA $A_{\text {TtE. In- }}$ terestingly, AVA ${ }_{c c T}$ was not correlated with AVA $_{T T E}$ in group with LVOT eccentricity $\geq 0.78$. 
Table 4. Subgroup analysis of the correlation among AVAs measured by CCT and TTE

\begin{tabular}{|c|c|c|}
\hline & Pearson's $r$ & $\mathrm{p}$-value \\
\hline \multicolumn{3}{|l|}{ Overall (127) } \\
\hline \multicolumn{3}{|l|}{ LVEF } \\
\hline$<50 \%(38)$ & 0.04 & 0.82 \\
\hline$\geq 50 \%(89)$ & 0.39 & $<0.001$ \\
\hline \multicolumn{3}{|l|}{ AVC Agatston score } \\
\hline$\leq 1651(103)$ & 0.31 & 0.00 \\
\hline$>1651(24)$ & 0.17 & 0.44 \\
\hline \multicolumn{3}{|l|}{ LVOT Eccentricity } \\
\hline$<0.78(58)$ & 0.40 & 0.002 \\
\hline$\geq 0.78(69)$ & 0.14 & 0.26 \\
\hline \multicolumn{3}{|l|}{ Atrial fibrillation } \\
\hline Yes (54) & 0.04 & 0.76 \\
\hline No (73) & 0.37 & 0.001 \\
\hline \multicolumn{3}{|l|}{ AVC Grade } \\
\hline Significant (98) & 0.39 & $<0.001$ \\
\hline Insignificant (29) & 0.35 & 0.17 \\
\hline \multicolumn{3}{|l|}{$\begin{array}{l}\text { Annular Calcification } \\
\text { Grade }\end{array}$} \\
\hline Significant (41) & 0.33 & 0.00 \\
\hline Insignificant (86) & 0.23 & 0.02 \\
\hline \multicolumn{3}{|l|}{ Transvalvular Mean PG } \\
\hline$>40$ mmHg (82) & 0.34 & 0.002 \\
\hline$\leq 40 \mathrm{mmHg}(45)$ & 0.04 & 0.78 \\
\hline
\end{tabular}

AVA = aortic valve area; $A V C$ = aortic valve calcification; $C C T$ = cardiac computed tomography; LVEF = left ventricular ejection fraction; LVOT = left ventricular outflow track; $P G=$ pressure gradient; $T T E=$ transthoracic echocardiography

CCT allows for accurate detection, localization, and quantification of calcification of the aortic valve and annulus [23]. We adopted a threshold of 1,651 Agatston score which correctly differentiated patients with severe AS from non-severe AS in the setting of low-flow grade AS [24]. Our result was consistent with a previous study showing that numeric difference between AVAcct and AVA $A_{T T E}$ was reduced with increasing Agatston score [15]. AVAcct was not correlated with AVA $_{\text {TTE }}$ in group with Agatston aortic valve score $>$ 1,651 . High Agatston aortic valve score results in dif- ficulty in drawing inner margin of aortic valve cusps because of blooming artifact from severe calcification, which can lead to inaccurate measurement that may contribute to under- or over- estimation of AVA when compared to absent or low Agatston aortic valve calcium score. We also found that the qualitative degree of aortic valve and annular calcification was not associated with difference between AVAccT and AVA $_{\text {TTE }}$ and AVA cct $_{\text {w }}$ was not correlated with AVA $A_{T T E}$ in group with absence or mild grade of aortic valve calcification. These results may indicate that quantitative assessment of aortic valve calcification on CCT is a factor that affects the discrepancy between AVA and AVAccr.

Of the two TTE parameters, only transvalvular mean pressure gradient was inversely associated with difference between AVAcct and AVA $A_{T T E}$ in a simple linear regression analysis. There was no correlation between AVA $_{\text {CCT }}$ and AVA $_{\text {TTE }}$ in patients with LVEF $<50 \%$ or with transvalvular pressure gradient $\leq 40 \mathrm{mmHg}$. These results do not explain why TTE parameters might have contributed to correlation between AVAcct and AVA $_{\text {TtE. }}$ However, the low flow state in which AS severity is overestimated due to incomplete opening of the calcified aortic valve might result in no correlation between AVAcct and AVATTE in severe AS [20].

AF is common in patients with AS [24]. In this study, $43 \%$ of patients had AF. AF may hamper precise measurement of aortic valve hemodynamics on TTE and deteriorate CCT image quality for the assessment of AVA due to mis-registration artifacts related to inconsistent RR intervals [26]. With the use of dual-source $C T$, diagnostic image quality was obtained for all patients, even for patients with AF. AF was not associated with difference between AVAcct and AVATTE in a simple linear regression analysis. In the subgroup analyses, it was shown that the correlation between AVAs measured by CCT and TTE varied significantly according to presence/absence of AF. As expected, there was no correlation between AVAcct and AVATTE in patients with AF.

Age showed a weak inverse association with difference between AVA сcт $_{\text {and }}$ AVA $A_{\text {TTE. }}$. This is consistent with previous study [15]. The evaluation of AS in the elderly may be difficult not only because of underlying diseases and clinical conditions, but also because of insufficient compliance with imaging testing. 
TTE is the first-line imaging modality for evaluation of AS severity. However, heterogeneous hemodynamic presentation, measurement errors, and ellipsoidal LVOT may influence the diagnosis and treatment decision for patients with severe AS when TTE is used. A recent study demonstrated that AVA measured by CCT correlated well with AVA assessed by TTE and catheter examination in 100 patients with severe calcified AS regardless of gender, presence of AF and heart rate [26]. Based on the our results which are in line with previous reports [9-15], aortic valve calcium score and LVOT area appeared to be the main factors significantly associated with difference between AVA cct $_{\text {and }}$ AVA $A_{T T E}$ in patients with severe AS and so they may be used to corroborate AS severity in case of discordant findings or poor acoustic windows at TTE. In addition, several factors such as TTE parameters, aortic valve and annular calcification, LVOT eccentricity, and AF need to be considered when comparing AVAs obtained with CCT and TTE. A combined approach using TTE and CCT might have incremental value over TTE alone for the evaluation of AS severity.

The present study has several limitations. First, we observed instances for which the difference between AVA сcт $_{\text {and }}$ AVA $A_{\text {TTE }}$ was high. However, we do not know which method is more accurate. There is no established non-invasive reference standard for assessment of AVA. Furthermore, there was no invasive reference obtained in this study. Second, the hemodynamic burden associated with the presence of AS is represented by the effective AVA and not the anatomic AVA. These points considerably limit the interpretation of the current findings. Third, the positioning at the edge of the aortic valve cusps for AVA planimetry by CCT can also generate some discrepancy. This was pointed out by the relative low intra-class coefficient for inter-observer AVA measurements. Fourth, 31\% and $19 \%$ of patients had moderate or greater mitral and aortic valve regurgitation. In addition, LVEF was diverse between $10 \%$ and $73 \%$ in this group. These factors would have a significant impact on flow profiles in both the LVOT and through the aortic valve that would compromise the accuracy of a continuity equation derived AVA compared to direct measurement through CCT. Finally, this was a single-institution retrospective study with a relatively small number of highly selected patients who had severe AS and underwent TAVR. This biases towards an older severe AS population with high surgical risk.

In conclusion, in patients being evaluated for TAVR with severe AS the mean AVA $A_{c c}$ was significantly larg-

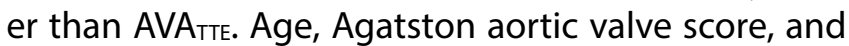
LVOT area difference between CCT and TTE might affect the difference between AVAcct and AVA $A_{\text {TTE }}$ in patients with severe AS. The clinical implications of this discrepancy are unknown and should be an area for future research.

\section{Conflict of Interest}

The authors have no conflict of interest relevant to this publication.

\section{Comment on this Article or Ask a Question}

\section{References}

1. Nkomo VT, Gardin JM, Skelton TN, Gottdiener JS, Scott CG, Enriquez-Sarano $M$. Burden of valvular heart diseases: a population-based study. Lancet. 2006;368:1005-1011. DOI: 10.1016/s01406736(06)69208-8

2. lung B, Vahanian A. Epidemiology of valvular heart disease in the adult. Nature Reviews Cardiology. 2011;8:162-172. DOI: 10.1038/nrcardio.2010.202

3. Webb JG, Wood DA. Current status of transcatheter aortic valve replacement. J Am Coll Cardiol. 2012;60:483-492. DOI: 10.1016/j.jacc.2012.01.071

4. Bilen $E$, Sari $C$, Durmaz $T$, Keleş $T$, Bayram $N A$, Akçay $M$, et al. The importance of echocardiography in transcatheter aortic valve implantation. Echocardiography. 2014;31:101-110. DOI: 10.1111/echo.12369

5. Kasel AM, Cassese S, Bleiziffer S, Amaki M, Hahn RT, Kastrati A, Sengupta PP. Standardized imaging for aortic annular sizing: implications for transcatheter valve selection. JACC Cardiovasc Imaging. 2013;6:249-262. DOI: 10.1016/j.jcmg.2012.12.005

6. Salgado RA, Leipsic JA, Shivalkar B, Ardies L, Van Herck PL, Op de Beeck BJ, et al. Procedural CT evaluation of transcatheter aortic valve replacement: what the radiologist needs to know. Radiographics. 2014;34:1491-1514. DOI: 10.1148/ rg.346125076
7. Mack MJ, Holmes DR, Webb J, Cribier A, Kodali SK, Williams MR, et al. Patient selection for transcatheter aortic valve replacement. J Am Coll Cardiol. 2013;62(17 Suppl):S1-10. DOI: 10.1016/j.jacc.2013.08.707

8. Saikrishnan N, Kumar G, Sawaya FJ, Lerakis $S$, Yoganathan AP. Accurate assessment of aortic stenosis: a review of diagnostic modalities and hemodynamics. Circulation. 2014;129:244-253. DOI: 10.1161/circulationaha.113.002310

9. Clavel MA, Malouf J, Messika-Zeitoun D, Araoz PA, Michelena HI, Enriquez-Sarano $M$. Aortic valve area calculation in aortic stenosis by $\mathrm{CT}$ and Doppler echocardiography. JACC Cardiovasc Imaging. 2015;8:248- 
257. DOI: $10.1016 /$ j.jcmg.2015.01.009

10. Pouleur AC, le Polain de Waroux JB, Pasquet A, Vanoverschelde JL, Gerber BL. Aortic valve area assessment: multidetector CT compared with cine MR imaging and transthoracic and transesophageal echocardiography. Radiology. 2007;244:745754. DOI: $10.1148 /$ radiol.2443061127

11. Utsunomiya $\mathrm{H}$, Yamamoto $\mathrm{H}$, Horiguchi J, Kunita E, Okada T, Yamazato R, et al. Underestimation of aortic valve area in calcified aortic valve disease: effects of left ventricular outflow tract ellipticity. Int J Cardiol. 2012;157:347-353. DOI: 10.1016/j. ijcard.2010.12.071

12. O'Brien B, Schoenhagen P, Kapadia SR, Svensson LG, Rodriguez L, Griffin BP, et al. Integration of $3 \mathrm{D}$ imaging data in the assessment of aortic stenosis: impact on classification of disease severity. Circ Cardiovasc Imaging. 2011;4:566-573. DOI: 10.1161/circimaging.111.964916

13. De Vecchi C, Caudron J, Dubourg B, Pirot N, Lefebvre V, Bauer F, et al. Effect of the ellipsoid shape of the left ventricular outflow tract on the echocardiographic assessment of aortic valve area in aortic stenosis. J Cardiovasc Comput Tomogr. 2014;8:5257. DOI: 10.1016/j.jcct.2013.12.006

14. Anger T, Bauer V, Plachtzik C, Geisler T, Gawaz MP, Oberhoff $M$, et al. Non-invasive and invasive evaluation of aortic valve area in 100 patients with severe aortic valvestenosis: comparison of cardiac computed tomography with $\mathrm{ECHO}$ (transesophageal/ transthoracic) and catheter examination. J Cardiol. 2014;63:189-197. DOI: 10.1016/j. jjcc.2013.08.002

15. Larsen LH, Kofoed KF, Carstensen HG, Mejdahl MR, Andersen MJ, Kjaergaard J, et al. Aortic valve area assessed with 320-detector computed tomography: comparison with transthoracic echocardiography. Int J Cardiovasc Imaging. 2014;30:165-173. DOI: 10.1007/s10554-013-0295-6

16. American College of C, American Heart Association Task Force on Practice G, Society of Cardiovascular A, Bonow RO, Carabello BA, Chatterjee K, et al. ACC/AHA 2006 guidelines for the management of patients with valvular heart disease: a report of the American College of Cardiology/American Heart Association Task Force on Practice Guidelines (writing Committee to Revise the 1998 guidelines for the management of patients with valvular heart disease) developed in collaboration with the Society of Cardiovascular Anesthesiologists endorsed by the Society for Cardiovascular Angiography and Interventions and the Society of Thoracic Surgeons. J Am Coll Cardiol. 2006;48:e1-148. DOI: 10.1161/circulationaha.106.177303

17. Rivard AL, Bartel T, Bianco RW, O'Donnell KS, Bonatti J, Dichtl W, et al. Evaluation of aortic root and valve calcifications by multi-detector computed tomography. J Heart Valve Dis. 2009;18:662-670. PMID: 20099715

18. Willmann JK, Weishaupt D, Lachat M, Kobza R, Roos JE, Seifert B, et al. Electrocardiographically gated multi-detector row $\mathrm{CT}$ for assessment of valvular morphology and calcification in aortic stenosis. Radiology. 2002;225:120-128. DOI: 10.1148/radiol.2251011703

19. Barbanti M, Yang TH, Rodès Cabau J, Tamburino C, Wood DA, Jilaihawi $\mathrm{H}$, et al. Anatomical and procedural features associated with aortic root rupture during balloon-expandable transcatheter aortic valve replacement. Circulation. 2013;128:244-253. DOI: 10.1161/circulationaha.113.002947

20. Pibarot P, Dumesnil JG. Aortic stenosis suspected to be severe despite low gradients. Circ Cardiovasc Imaging. 2014;7:545-551. DOI: 10.1161/circimaging. 113.001375

21. Gaspar T, Adawi S, Sachner R, Asmer I, Ganaeem $M$, Rubinshtein $R$, et al. Three-dimensional imaging of the left ventricular outflow tract: impact on aortic valve area estimation by the continuity equation. J Am Soc Echocardiogr. 2012;25:749-757. DOI: 10.1016/j.echo.2012.05.001
22. Saitoh T, Shiota M, Izumo $M$, Gurudevan SV, Tolstrup K, Siegel RJ, et al. Comparison of left ventricular outflow geometry and aortic valve area in patients with aortic stenosis by 2-dimensional versus 3-dimensional echocardiography. Am J Cardiol. 2012;109:1626-1631. DOI: 10.1016/j.amjcard.2012.01.391

23. Koos R, Mahnken AH, Sinha AM, Wildberger JE, Hoffmann R, Kuhl HP. Aortic valve calcification as a marker for aortic stenosis severity: assessment on 16-MDCT. AJR Am J Roentgenol. 2004;183:1813-1818. DOI: 10.2214/ajr.183.6.01831813

24. Cueff C, Serfaty JM, Cimadevilla C, Laissy JP, Himbert D, Tubach F, et al. Measurement of aortic valve calcification using multislice computed tomography: correlation with haemodynamic severity of aortic stenosis and clinical implication for patients with low ejection fraction. Heart. 2011;97:721726. DOI: $10.1136 /$ hrt.2010.198853

25. Greve AM, Gerdts E, Boman K, Gohlke-Baerwolf $C$, Rossebø $A B$, Nienaber $C A$, et al. Prognostic importance of atrial fibrillation in asymptomatic aortic stenosis: The Simvastatin and Ezetimibe in Aortic Stenosis study. Int J Cardiol. 2013;166:72-76. DOI: 10.1016/j.ijcard.2011.09.064

26. Burup Kristensen $C$, Jensen JS, Sogaard $P$, Carstensen HG, Mogelvang R. Atrial fibrillation in aortic stenosis--echocardiographic assessment and prognostic importance. Cardiovasc Ultrasound. 2012;10:38. DOI: 10.1186/1476-7120-10-38

Cite this article as: Ko SM, Condado JF, Babaliaros V, Lerakis S, Ko Y, Stillman AE. Difference in Aortic Valve Area Measured With Cardiac CT and Transthoracic Echocardiography. Structural Heart Disease. 2019;5(3):52=61. DOI: https:// doi.org/10.12945/j.jshd.2019.025.18 